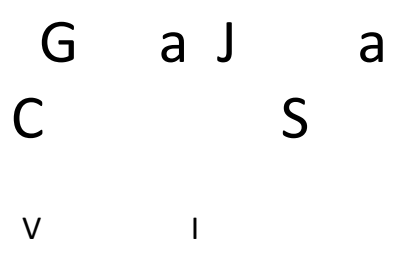

(U) Global Journal of

www.awer-center/gjcs

\title{
Automated detection of optic disc in retinal fundus images using gabor filter kernels
}

\begin{tabular}{|c|c|c|c|c|c|c|c|c|}
\hline Baha Sen*, Fa & $E$ & $\mathrm{Na}$ & a $S$ & $Y$ & B & U & & a a $\mathrm{T}$ \\
\hline Kemal Akyol, E & $\mathrm{Fa}$ & Ka a & $U$ & $\mathrm{Ba}$ & a a & $\mathrm{Ka} \mathrm{a}$ & $\mathrm{T}$ & \\
\hline Safak Bayir, E & $\mathrm{Fa}$ & $\mathrm{Ka} \mathrm{a}$ & $U$ & $\mathrm{Ba}$ & a a a & $\mathrm{Ka} \mathrm{a}$ & $\mathrm{T}$ & \\
\hline Hilal Kaya, Fa & $E$ & $\mathrm{Na}$ & a $S$ & $Y$ & B & $U$ & & a a $\mathrm{T}$ \\
\hline
\end{tabular}

Suggested Citation:

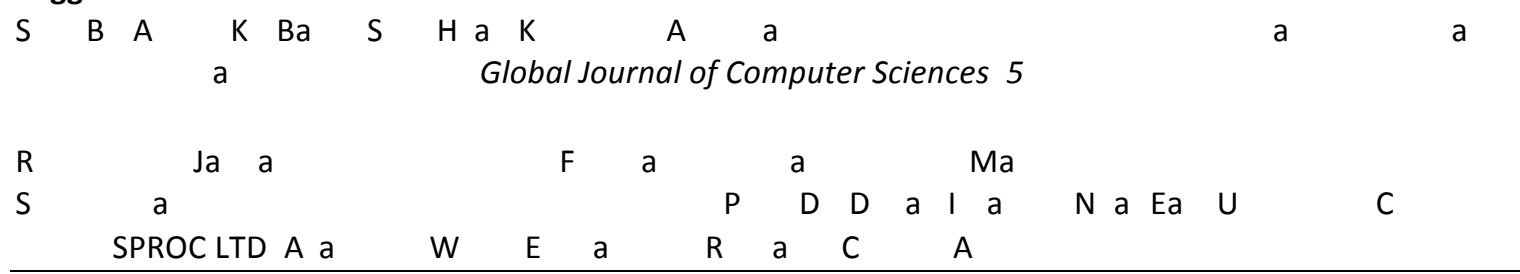

\section{Abstract}

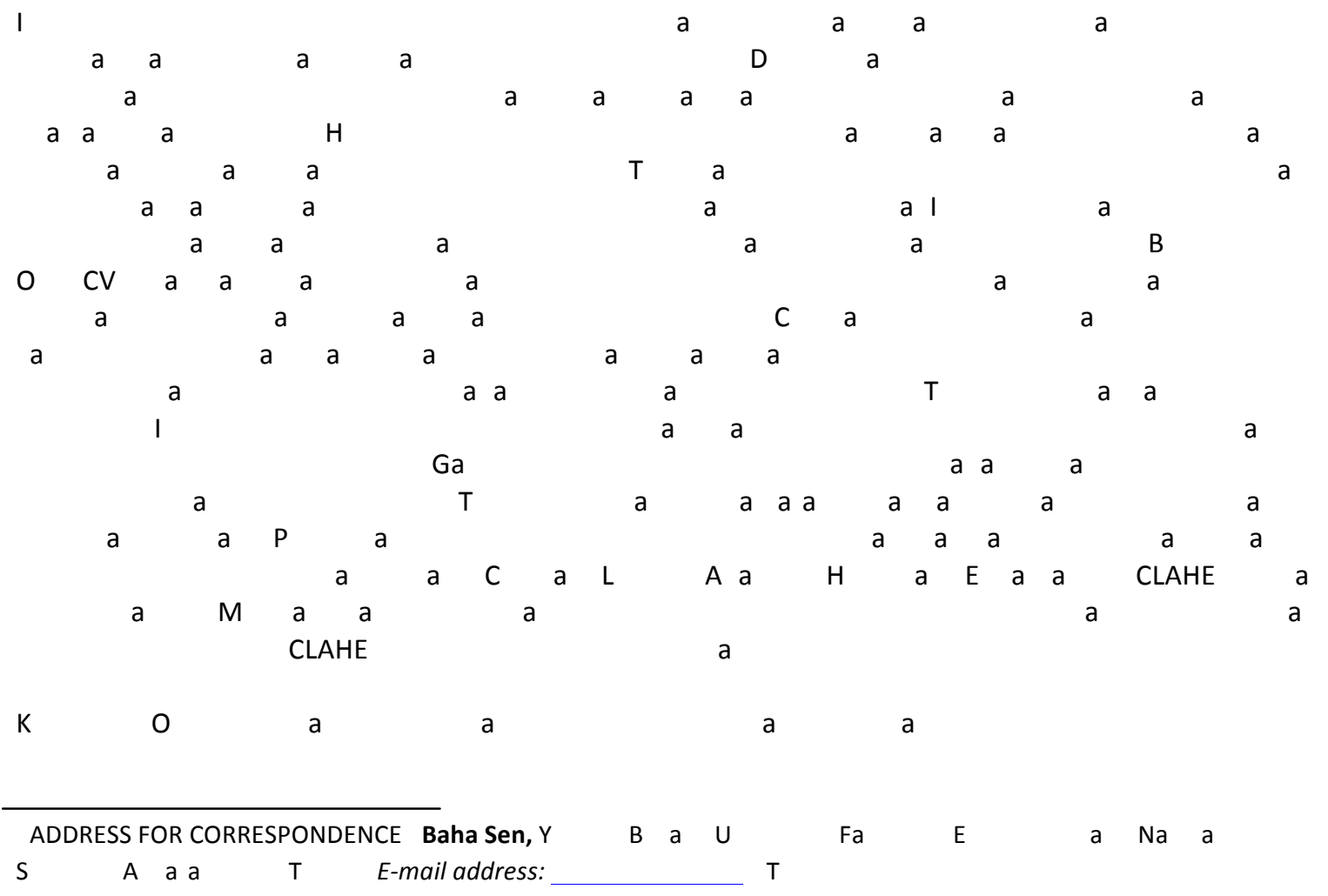


$\begin{array}{lllllllllll}\mathrm{S} & \mathrm{B} & \mathrm{A} & \mathrm{K} & \mathrm{Ba} & \mathrm{S} & \mathrm{H} & \mathrm{a} & \mathrm{K} & \mathrm{A} & \mathrm{a}\end{array}$

Global Journal of Computer Sciences 5

\section{Introduction}

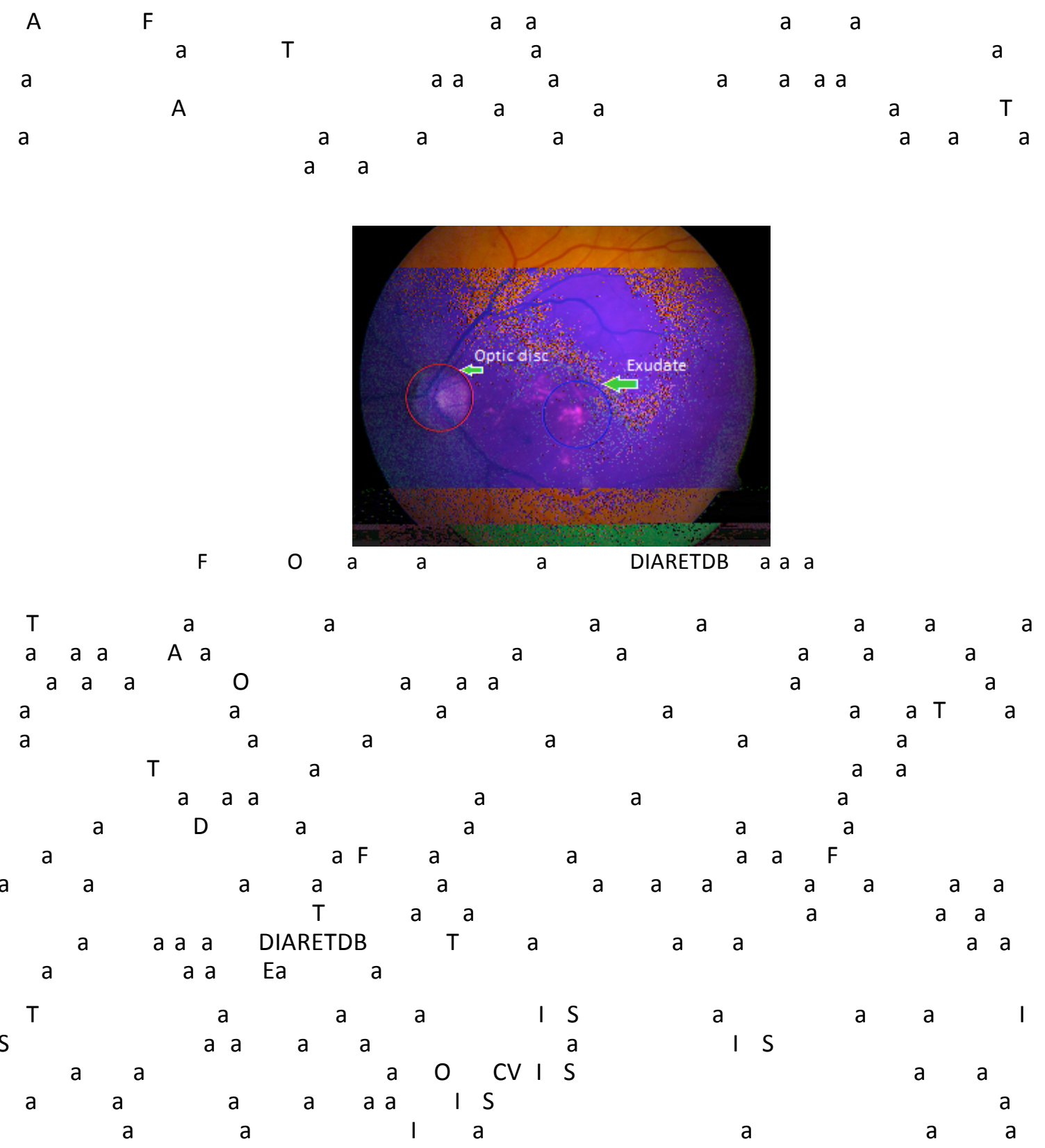

\section{Related Works}

T a a

B

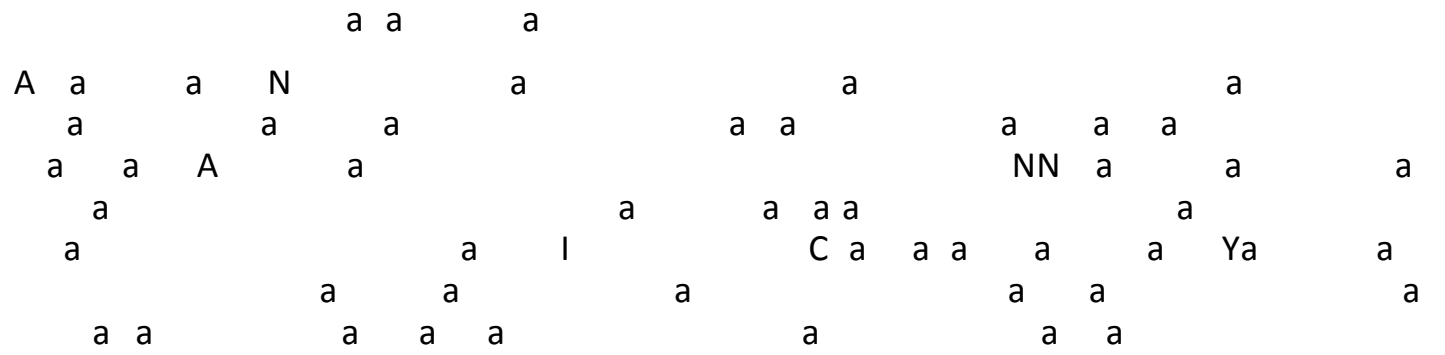




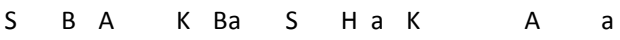

Global Journal of Computer Sciences 5

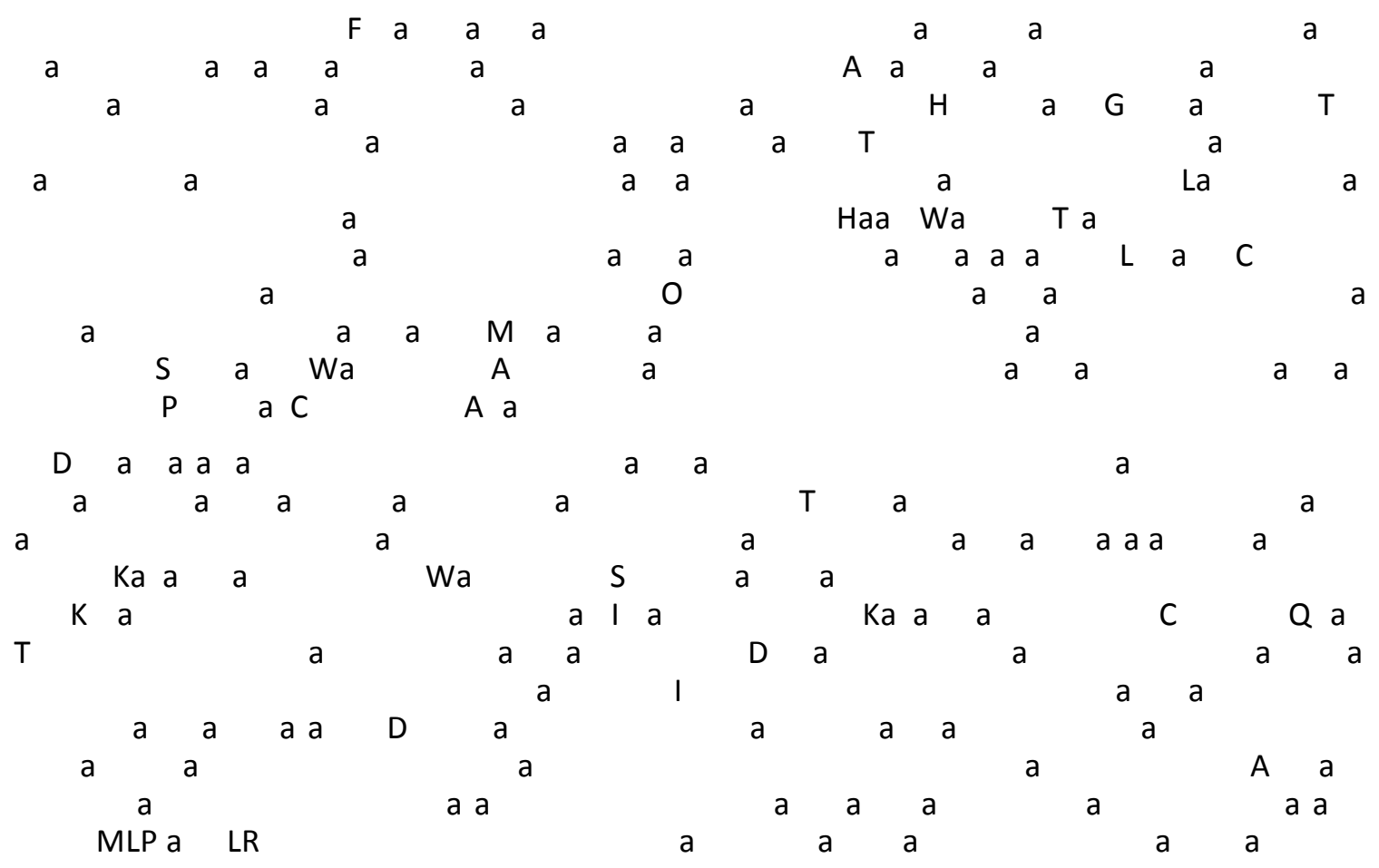

\section{Texture Analysis}

$\mathrm{T}$

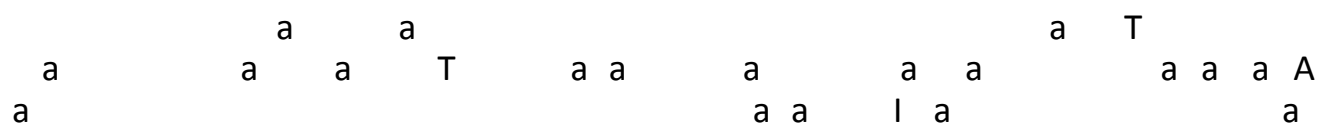

a

a a a a

a a I a

a

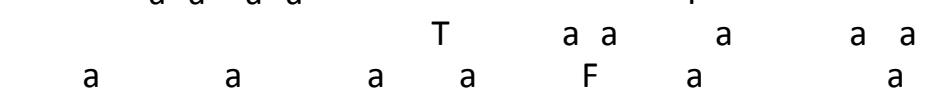

a

a $\quad a^{a} \quad a^{a} \quad a^{a} a$

a

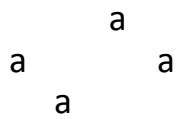

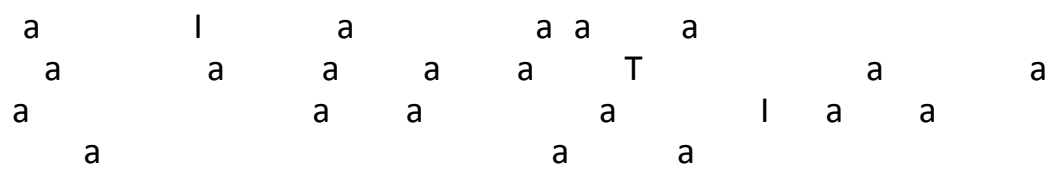

Ga $\quad$ a $\quad a \quad a$

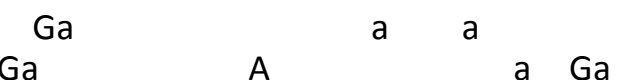

a

$\begin{array}{lllll}a & a & a & & a \\ a^{n} & & a^{2}\end{array}$
a

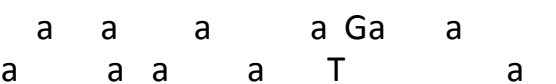
I
a $a \quad$ a $a$
a $a$
$\varphi(x, y)=\frac{F^{2}}{\pi \gamma n} e^{-F^{2}\left[\left(\frac{x^{\prime}}{\gamma}\right)^{2}+\left(\frac{y^{\prime}}{n}\right)^{2}\right]} e^{i 2 \pi F x^{\prime}} ;$

$\left\{\begin{array}{c}x^{\prime}=x \cos \theta+y \sin \theta \\ y^{\prime}=-x \sin \theta+y \cos \theta\end{array}\right.$ 
$\begin{array}{llllllllllllllllll}\mathrm{S} & \mathrm{B} & \mathrm{A} & \mathrm{K} & \mathrm{Ba} & \mathrm{S} & \mathrm{H} & \mathrm{a} & \mathrm{K} & \mathrm{A} & \mathrm{a}\end{array}$

Global Journal of Computer Sciences 5

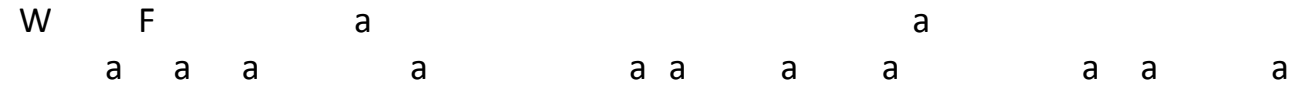

$$
\begin{aligned}
& \begin{array}{rrrrr}
a & a & a & a & a
\end{array} \\
& \begin{array}{lllll} 
& a & a & a & a \\
& a & a & a
\end{array} \\
& \begin{array}{lllllllll}
a & A & & & a & & a & a & a \\
a & & a & a & & a & a & & a
\end{array} \\
& \text { a } \mathrm{Ga} \quad \mathrm{a} \quad \mathrm{a} \\
& \varphi(u, v)=e^{-\frac{\pi^{2}}{F^{2}}\left[\gamma^{2}\left(u^{\prime}-F\right)^{2}+n^{2} v^{\prime 2}\right]} \\
& \left\{\begin{array}{c}
u^{\prime}=u \cos \theta+v \sin \theta \\
v^{\prime}=-u \sin \theta+v \cos \theta
\end{array}\right.
\end{aligned}
$$

\section{Computer Vision and OpenCV}

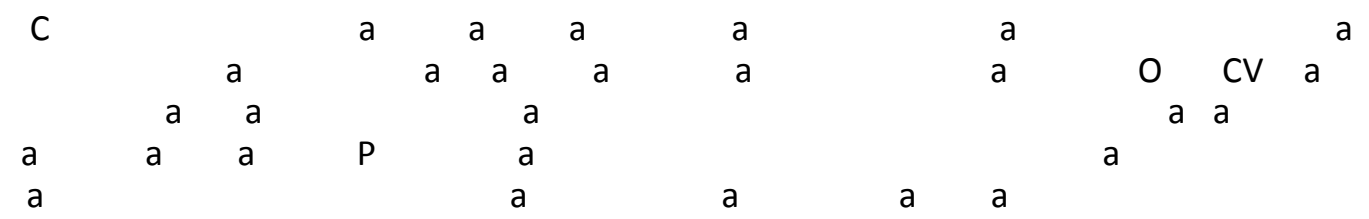

\section{Image Enhancement and Image Datasets}

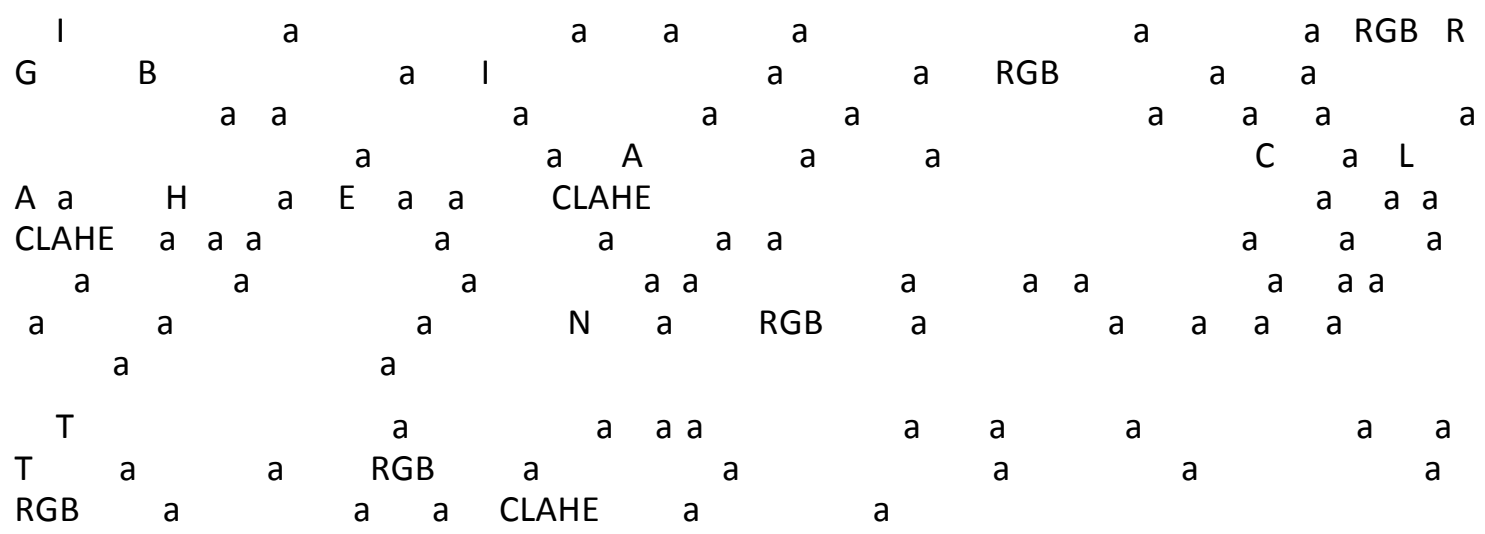

\section{Proposed Method}

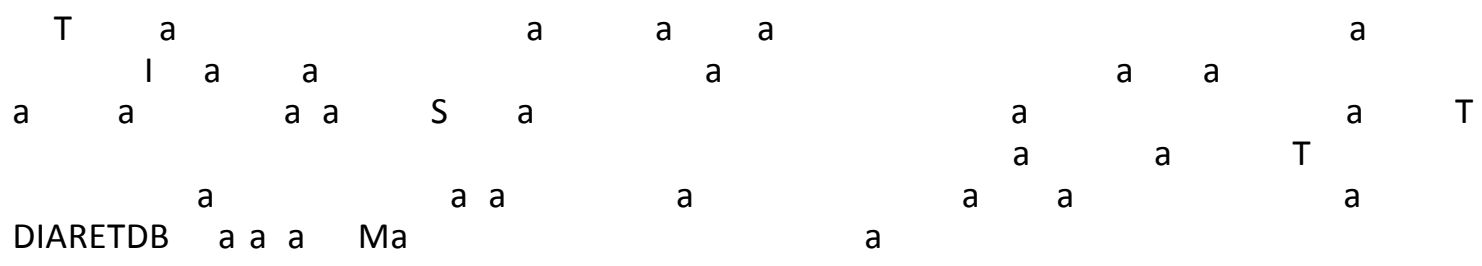




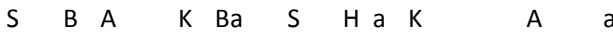

Global Journal of Computer Sciences 5

- P a a

$\bullet T$ a

a a

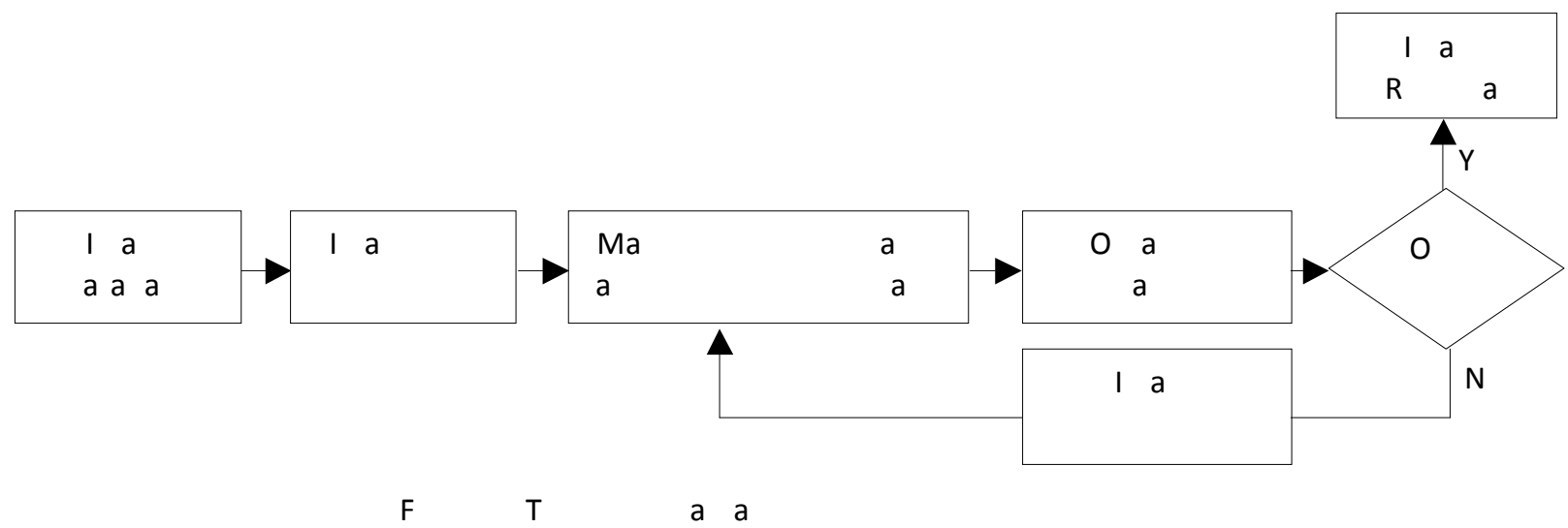

5.1. Preparation of the retinas

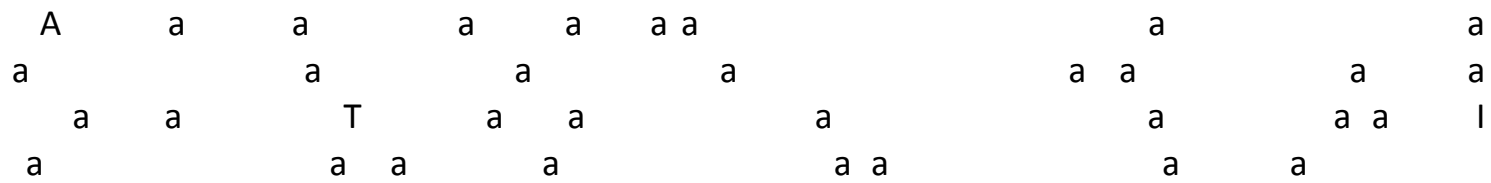

a

a a

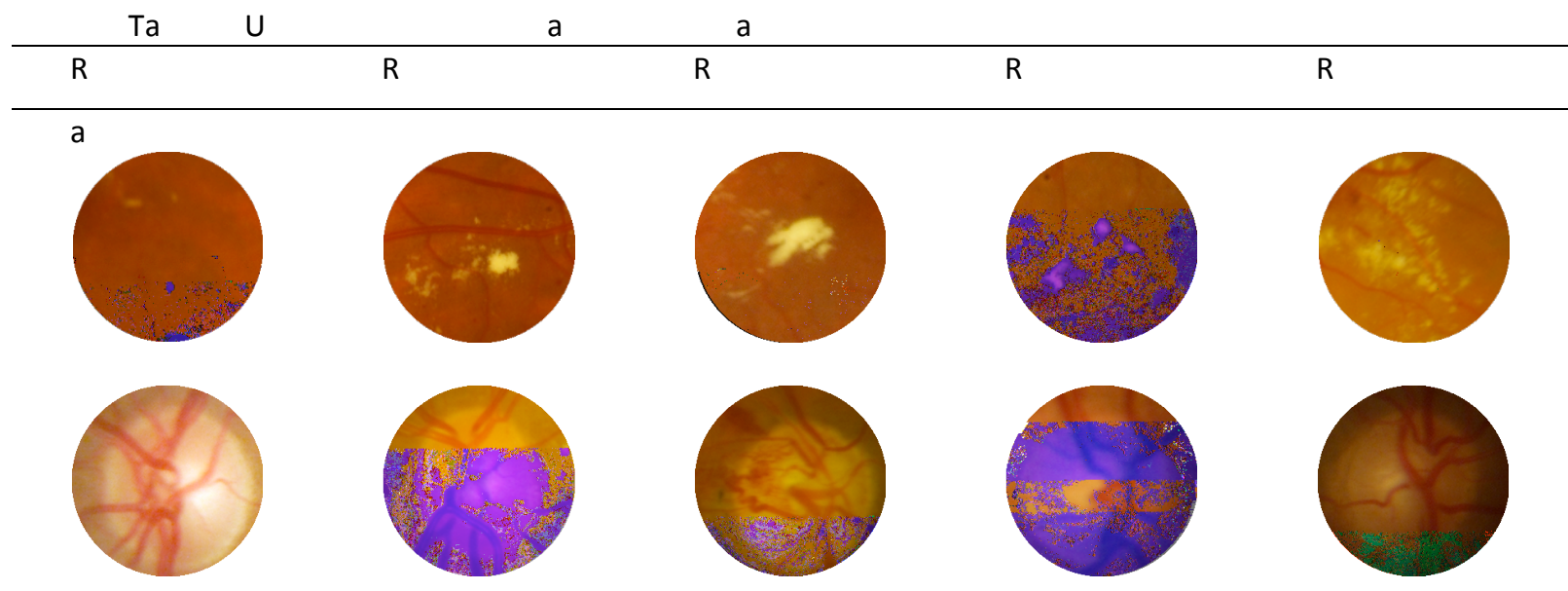

5.2. Testing regions with texture analysis
A
$a^{a} \quad a \quad a \quad \stackrel{a}{a}$
a $a$
a
a
A
a
a L
a a $a$ $\mathrm{O}_{\mathrm{a}}^{\mathrm{CV}} \mathrm{A}^{\mathrm{a}} \mathrm{a}$ a
a
$\begin{array}{ll}a & a \\ a & a\end{array}$
a $\quad$ a $\quad$ a
a $\quad$ a $\quad \begin{aligned} & a \\ & \quad\end{aligned} \quad a$
a a a
a L
a $\quad$ a $\quad$ a
a 
$\begin{array}{lllllllll}S & \text { B } & \text { A } & \text { K } & \text { Ba } & S & \mathrm{H} & \text { a } & \text { K }\end{array}$

Global Journal of Computer Sciences 5

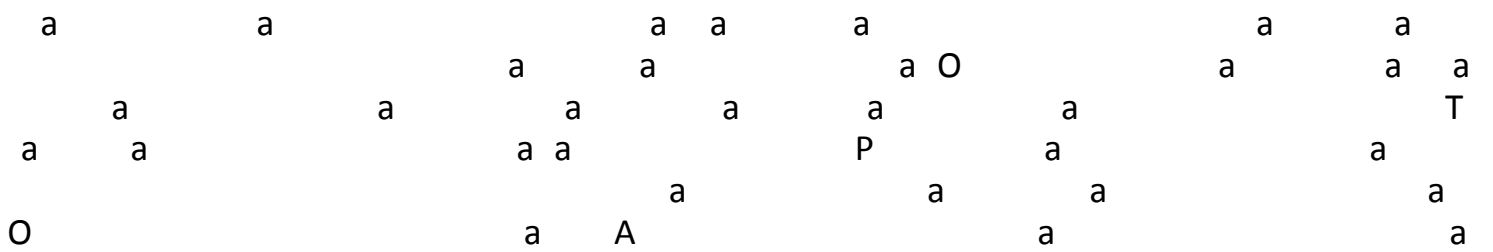

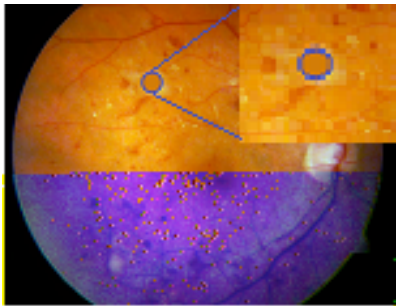

a S

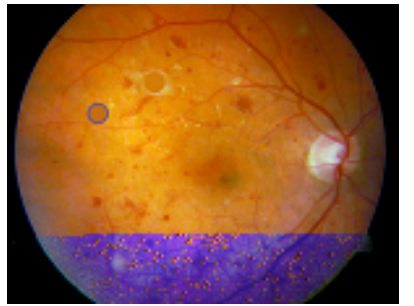

a S
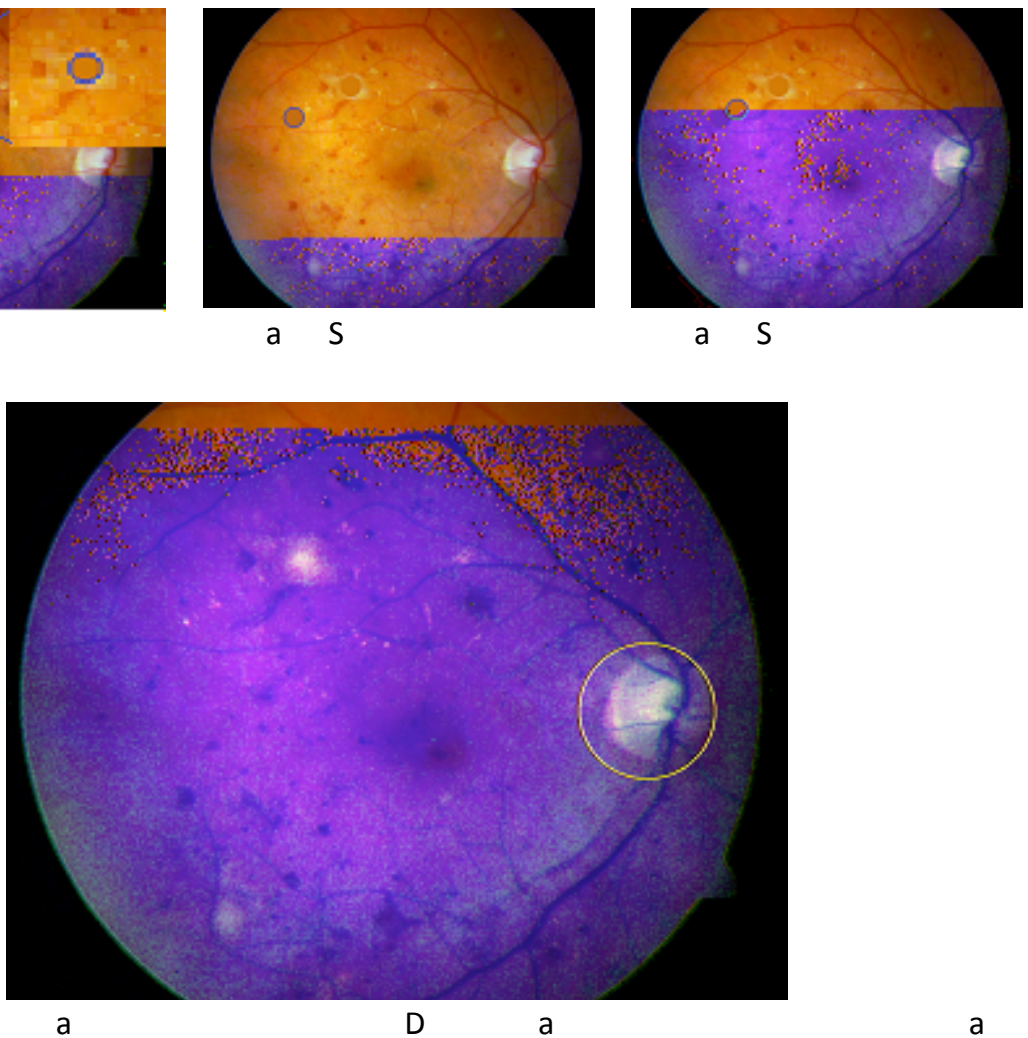

$\mathrm{F} \quad \mathrm{a} \mathrm{T}$

a a

$\begin{array}{lll}\text { W } & \text { a } & \text { a } \\ \mathrm{a} & & \end{array}$

a

a

a

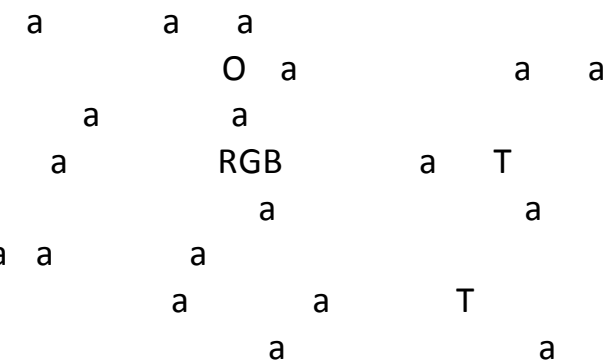

a a

CLAHE

a

a a a a


$\begin{array}{lllllllllllllllll}\mathrm{S} & \mathrm{B} & \mathrm{A} & \mathrm{K} & \mathrm{Ba} & \mathrm{S} & \mathrm{H} & \mathrm{a} & \mathrm{K} & \mathrm{A} & \mathrm{a}\end{array}$

Global Journal of Computer Sciences 5

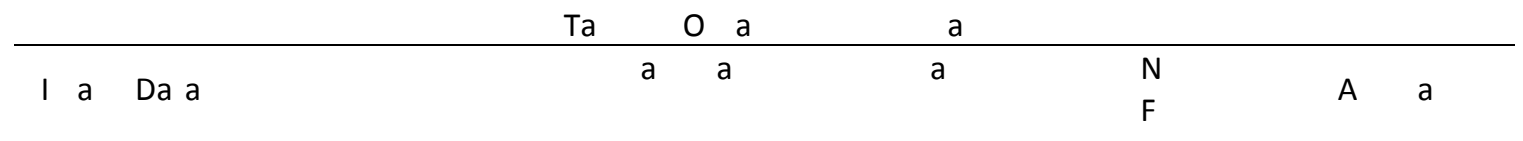

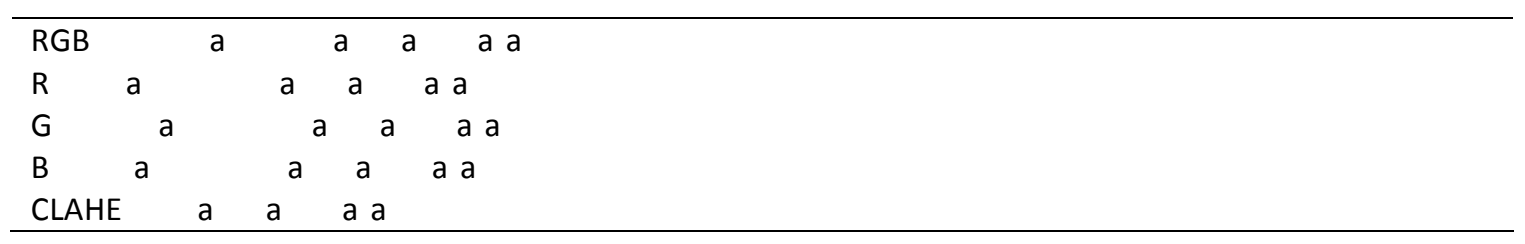

\section{Conclusion and Future Work}

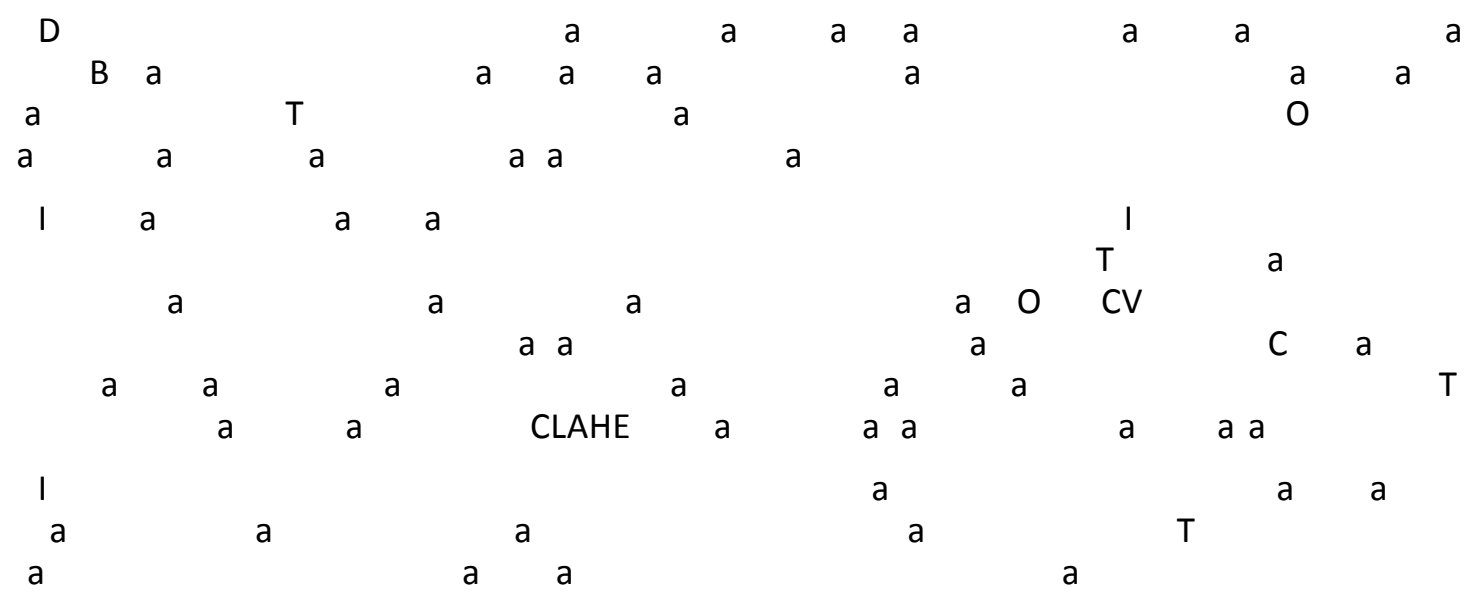

\section{Acknowledgements}
T a
DIARETDB
a
a
a a a

\section{References}

I a C Detection of Optic Disc and Macula and Their Evaluation in Retinal Fundus Images, Karadeniz Technical University Institute of Natural Sciences, Trabzon.
Ka J S a H P A a $\begin{array}{llllll}\text { a } & \text { a } & \text { a } & \text { a } & \text { a } & \text { a }\end{array}$ International Journal of Advanced Research in Computer Science and Software Engineering, 2(4), pp 242-249.
Ka T Ka $\quad$ V Ka a a JK L L S I Ra A V a $R$ P a J Ka a $\quad H \quad U$ a $H \quad$ Da $\quad$ Da $R$ a Da a a E a a
$P \quad$ Proceedings of the Medical Image Understanding and Analysis (MIUA 2007), Aberystwyth: UK.

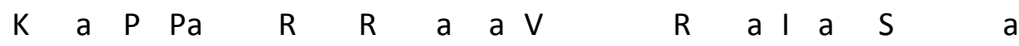 M International Journal of Computer Applications 86
$\begin{array}{lllllllllll}A & a & M & D & N & M & T & \text { a } & \text { a }\end{array}$ International Conference of the IEEE Engineering in Medicine and Biology Society, 1
$\mathrm{C}$ a a a T Y a a a S S Z Ta

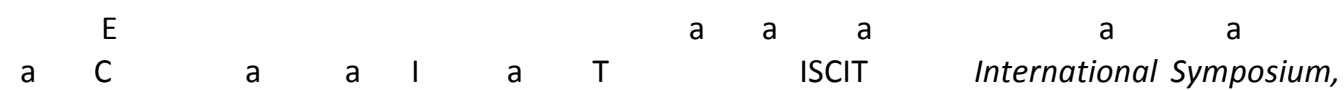




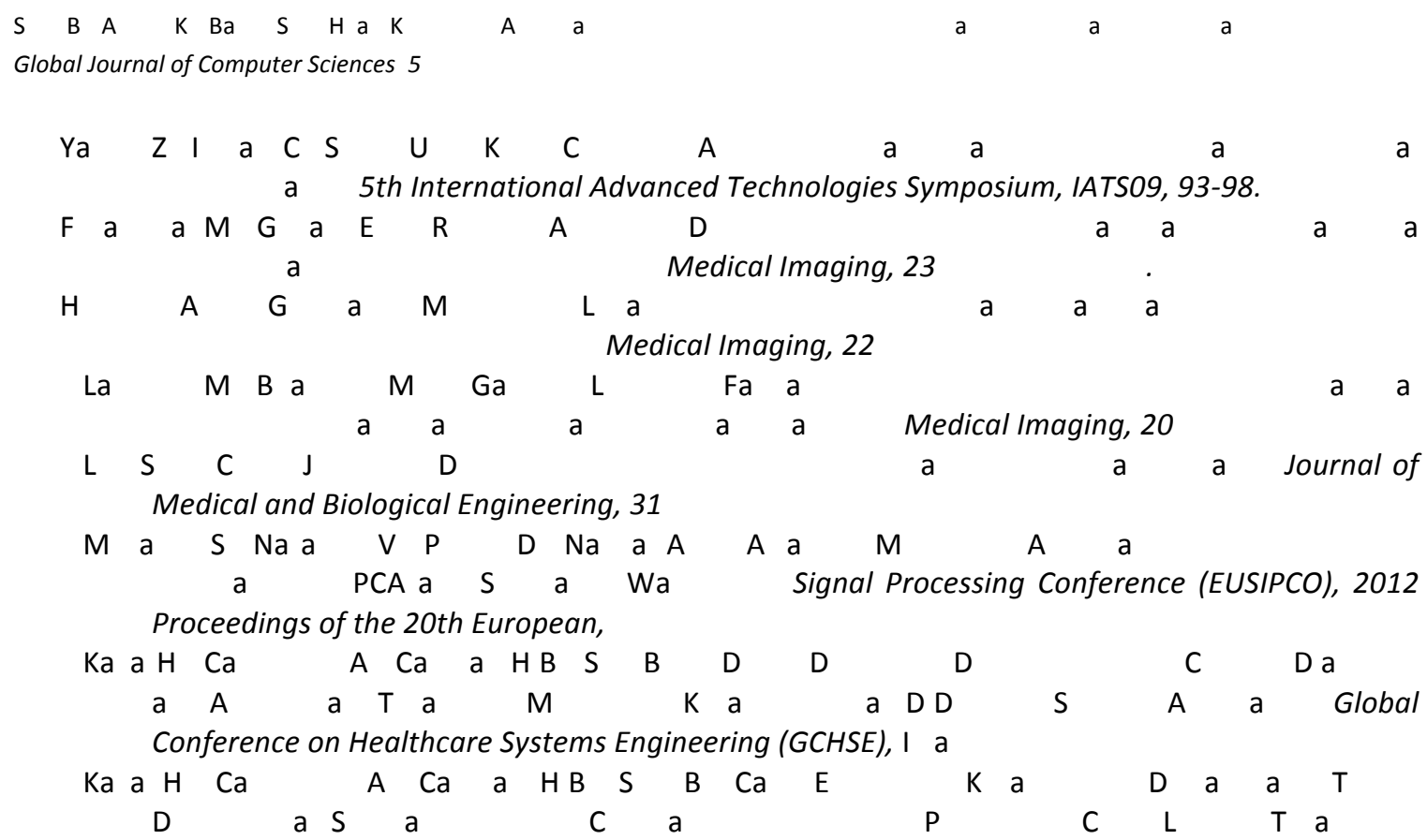

Emerging Trends in Computational Biology, Bioinformatics, and Systems Biology, Elsevier ISBN

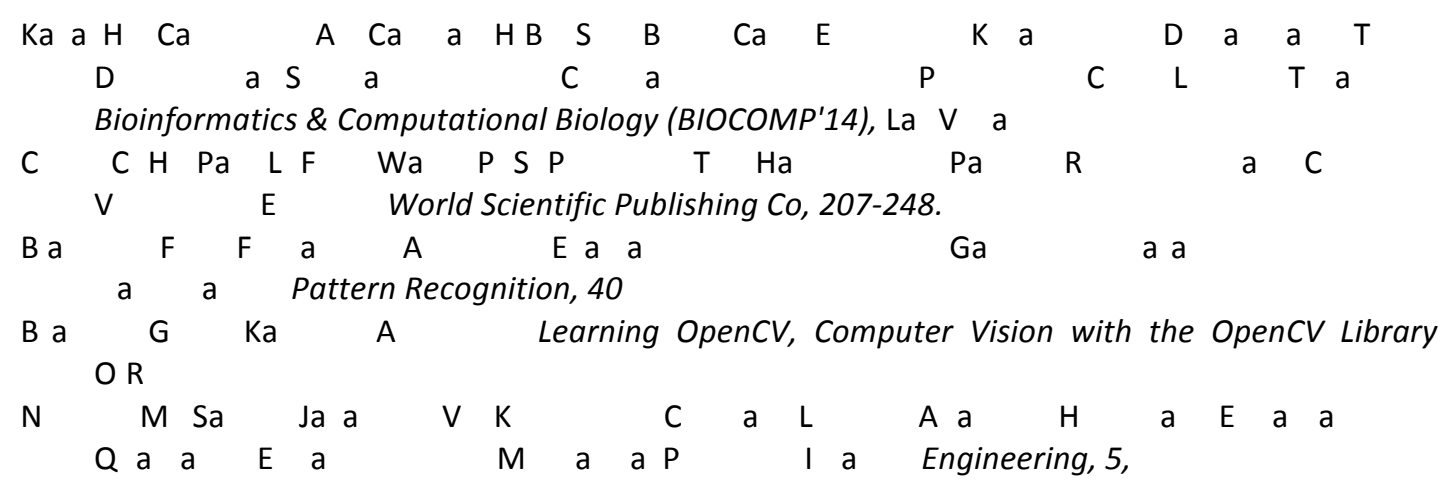

